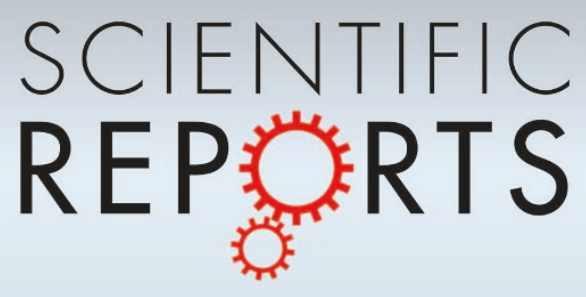

OPEN

SUBJECT AREAS:

ELECTRONIC DEVICES

ELECTRONIC PROPERTIES AND

MATERIALS

Received

30 October 2013

Accepted

16 January 2014

Published

4 February 2014

Correspondence and requests for materials should be addressed to

H.F. (hfujioka@iis. u-tokyo.ac.jp)

\section{Field-effect transistors based on cubic} indium nitride

\author{
Masaaki Oseki ${ }^{1}$, Kana Okubo' $^{1}$, Atsushi Kobayashi' ${ }^{1}$ Jitsuo Ohta' \& Hiroshi Fujioka ${ }^{1,2}$ \\ ${ }^{1}$ Institute of Industrial Science, The University of Tokyo, Tokyo 153-8505, Japan, ${ }^{2}$ CREST, Japan Science and Technology Agency, \\ Tokyo 102-0075, Japan.
}

\begin{abstract}
Although the demand for high-speed telecommunications has increased in recent years, the performance of transistors fabricated with traditional semiconductors such as silicon, gallium arsenide, and gallium nitride have reached their physical performance limits. Therefore, new materials with high carrier velocities should be sought for the fabrication of next-generation, ultra-high-speed transistors. Indium nitride (InN) has attracted much attention for this purpose because of its high electron drift velocity under a high electric field. Thick InN films have been applied to the fabrication of field-effect transistors (FETs), but the performance of the thick InN transistors was discouraging, with no clear linear-saturation output characteristics and poor on/off current ratios. Here, we report the epitaxial deposition of ultrathin cubic InN on insulating oxide yttria-stabilized zirconia substrates and the first demonstration of ultrathin-InN-based FETs. The devices exhibit high on/off ratios and low off-current densities because of the high quality top and bottom interfaces between the ultrathin cubic InN and oxide insulators. This first demonstration of FETs using a ultrathin cubic indium nitride semiconductor will thus pave the way for the development of next-generation high-speed electronics.
\end{abstract}

T heoretical calculations based on optical measurements predict that $\mathrm{InN}$ possesses a high electron peak velocity exceeding $2 \times 10^{8} \mathrm{~cm} / \mathrm{s}$ (ref. 1). This value is much higher than those for conventional semiconductor materials such as gallium arsenide, used in high-speed electronics. This nature of $\mathrm{InN}$ makes it a promising material for use in high-speed FETs. In fact, it is predicted that an InN-based metal-oxidesemiconductor FET (MOSFET) with a channel length of $100 \mathrm{~nm}$ can be theoretically operated at terahertz frequencies ${ }^{2}$. Despite these outstanding transport properties, the performance of FETs fabricated with thick $\mathrm{InN}(>20 \mathrm{~nm})$ has been discouraging ${ }^{3}$. The transistors show poor on/off ratios and lack clear linear-saturation output characteristics. It is believed that the problems with these InN transistors stem from the poor quality of the thick InN films and their interfaces with the surrounding insulators. InN films are typically grown on foreign crystals such as GaN (refs. 4 and 5) or AlN (refs. 3 and 6), resulting in quite large lattice mismatches, which in turn leads to the formation of a high density of misfit dislocations at the interfaces ${ }^{7}$. These dislocations then propagate into the InN films and act as unintentional sources of electron ${ }^{8,9}$, making it difficult to modulate the source-drain current $\left(I_{\mathrm{DS}}\right)$ in the FET via gating. Therefore, a new technique that enables the deposition of $\mathrm{InN}$ films with higher structural perfection must be developed for successful fabrication of high-performance InN-based FETs. The use of yttria-stabilized zirconia (YSZ) substrates can be a good option for this purpose because there is a very small lattice mismatch $(2.7 \%)$ between YSZ and InN, which should provide a reduced defect density at the interfaces ${ }^{10,11}$. The other advantage of using YSZ substrates lies in their insulating nature, which is important for the fabrication of FETs because the use of semiconducting substrates may lead to an increase in the leakage current in the "off" state.

We report a newly developed method for the growth of ultrathin cubic InN(111) films on YSZ(111) substrates and the first demonstration of transistors using high-quality ultrathin cubic $\mathrm{InN}$ channel layers. The transistors fabricated with a 2-nm-thick cubic film exhibited an on/off $I_{\mathrm{DS}}$ ratio of $\sim 5 \times 10^{6}$ and a very low off-current $I_{\mathrm{DS}}$ of the order of pA. The maximum $I_{\mathrm{DS}}$ for devices fabricated in this study exceeded $60 \mathrm{~mA} / \mathrm{mm}$ when flowing through a 5-nm-thick InN layer, and the field-effect mobility was $\sim 58 \mathrm{~cm}^{2} \mathrm{~V}^{-1} \mathrm{~s}^{-1}$.

\section{Results}

Prior to film growth, the YSZ(111) substrates were thermally treated at $1000^{\circ} \mathrm{C}$ in air so that InN films with atomically flat surfaces could grow on them. During annealing, the substrates were capped with a sapphire wafer. This capping step is essential for reproducibly obtaining high-quality $\mathrm{InN}$ films on YSZ. Figures $1 \mathrm{a}$ and $\mathrm{lb}$ show atomic force microscopy (AFM) images of YSZ(111) substrates before and after the thermal treatment. The 
a

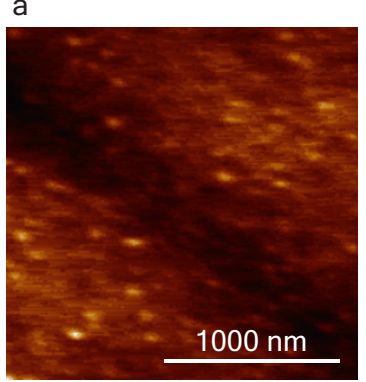

b

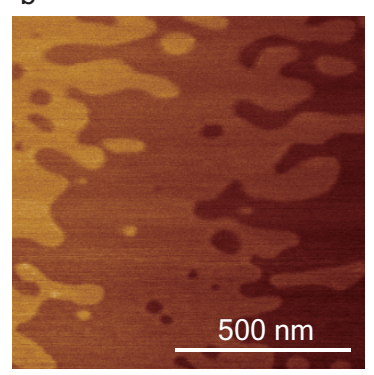

C

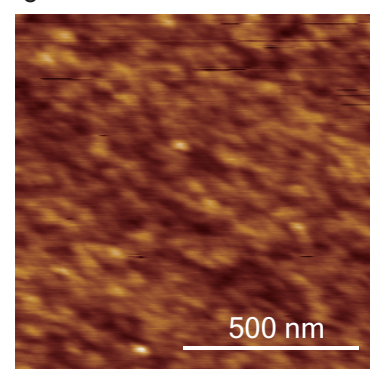

Figure $1 \mid$ AFM surface images of YSZ(111) and ultrathin InN. (a) As-received YSZ(111) surface. (b) The surface of YSZ became atomically flat after thermal annealing at $1000^{\circ} \mathrm{C}$. Atomic steps appeared on the surface. (c) Epitaxial InN film grown on the atomically flat YSZ(111). The root mean square roughness for the $\mathrm{InN}$ surface is $0.18 \mathrm{~nm}$.

annealed surface possesses a clear step-and-terrace structure with a single step height of $\sim 0.6 \mathrm{~nm}$, which roughly corresponds to two monolayers of YSZ(111). The cross-sectional profile of the atomically flat YSZ(111) surface is provided in Supplementary Fig. S1b. Atomically flat surfaces are critical for epitaxial growth of high-quality InN films and the fabrication of transistors because surface roughness often leads to generation of defects.

InN films were grown at $620^{\circ} \mathrm{C}$ via pulsed-sputtering deposition in an ultra-high vacuum chamber with a background pressure of $\sim 2 \times$ $10^{-10}$ Torr (refs. 11 and 12). An In metal target and purified nitrogen gas (99.9999\% purity) were used as the sources for InN growth. The InN film thickness was controlled by varying the pulse number during sputtering. The average deposition rate of the film precursors was adjusted so that growth of an InN monolayer was completed in $2.7 \mathrm{~s}$. The growth temperature is from a thermo-couple that locates near the substrate, which indicates that the actual temperature of the growing surface is slightly lower than that value. The epitaxial InN films formed on the YSZ(111) substrates are continuous and without cracks or pits on the surface (Fig. 1c). The root mean square value of the surface roughness for the films was as low as $0.18 \mathrm{~nm}$, which is smooth enough to fabricate an abrupt interface with, for example, a gate oxide insulator used in the fabrication of FETs.

Figure 2a shows a cross-sectional transmission electron microscopic (TEM) image of ultrathin (2 $\mathrm{nm}$ ) InN grown on a YSZ(111) substrate. Because the sample is part of FET, an amorphous $\mathrm{HfO}_{2}$ gate insulator is deposited on top of the $\mathrm{InN}$ film. It is well known that the stacking sequences of hexagonal (wurtzite) InN along the $<0001>$ direction and cubic (zincblende) InN along the $<111>$ direction are ..ABABAB.. and ...ABCABC.., respectively, where $A, B$, and $C$ denote In-N layers with different atom positions. Careful interpretation of the TEM image revealed that the stacking sequence of the InN is that of the (111)-oriented cubic (zincblende) structure. In addition, stacking sequence of the cubic InN replicated that of the YSZ substrate. The successful growth of cubic InN is likely because of the stacking sequence of fluorite structure in YSZ, which has a zincblende-like structure. White dots in the YSZ in the figure are $\mathrm{Zr}(\mathrm{Y})$ atoms stacked in the sequence ..ABCABC.., which is the same as that for cubic InN. Figures $2 \mathrm{~b}$ and $2 \mathrm{c}$ show the Fourier transformed images of the InN and YSZ in Fig. 2a, respectively. The distribution of spots in Fig. 2b is quite similar to that of Fig. 2c, which indicates that the InN crystallized with the cubic structure. All these results support the conclusion that the growth of cubic $\operatorname{InN}(111)$ occurred on the cubic YSZ(111) substrates. Considering the successful growth of $\mathrm{N}$-polar InN(000-1) films at $615^{\circ} \mathrm{C}$ by plasma-assisted molecular beam epitaxy ${ }^{13}$, we believe that the cubic $\mathrm{InN}$ is as stable as $\mathrm{N}$-polar $\mathrm{InN}$, at this moment.

FETs with InN channel thicknesses of 2 and $5 \mathrm{~nm}$ were then fabricated. An optical microscopic image and schematic view of a FET are shown in Fig. 3. To fabricate the FETs, $100 \mu \mathrm{m} \times 50 \mu \mathrm{m}$ islands in the ultrarhin InN films on the YSZ(111) substrates were formed using photolithography and inductively coupled plasma reactive ion etching techniques. A 50-nm-thick Au layer was then deposited on each of the InN surfaces and used as source and drain electrodes. A 20 -nm-thick $\mathrm{HfO}_{2}$ gate insulator was then deposited at $200^{\circ} \mathrm{C}$ on each FET using atomic layer deposition. The $\mathrm{HfO}_{2}$ was in the amorphous phase, which was confirmed by analyzing the TEM
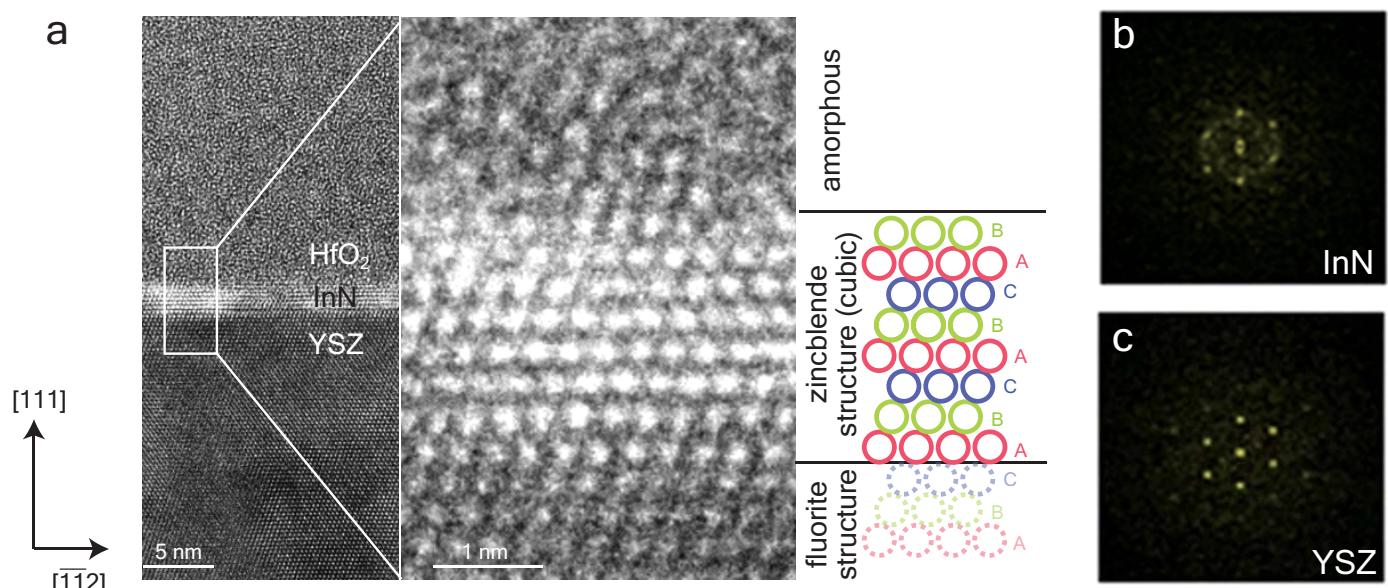

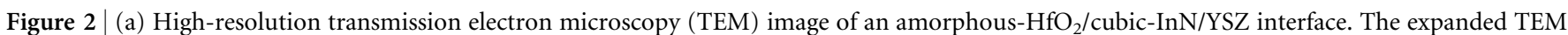

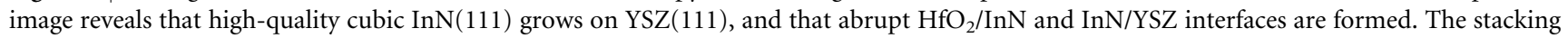
sequence for YSZ is the same as that for InN. (b) Fourier-transformed image of InN, (c) Fourier-transformed image of YSZ in the TEM image. The symmetries in (b) and (c) are the same, indicating that the symmetry of the cubic YSZ was transferred to the InN film. 

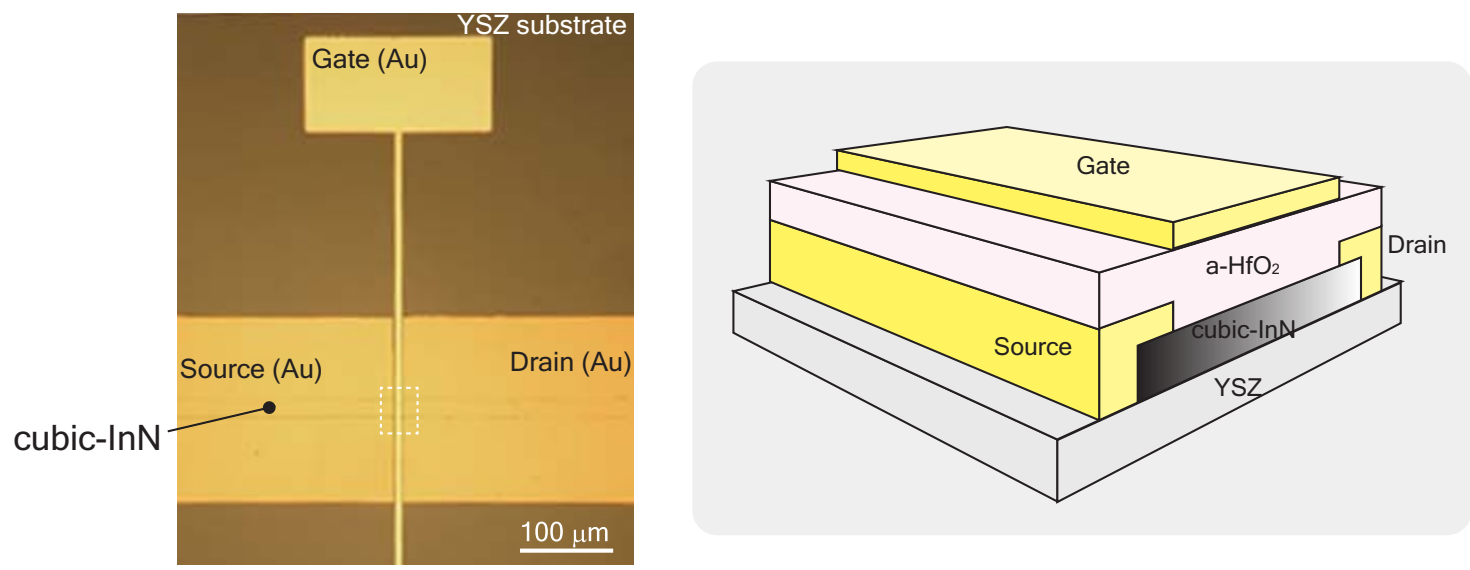

Figure 3 Optical micrograph and schematic image of a cubic (zincblende) InN-based field-effect transistor. Mesa etching of cubic InN epitaxially grown on YSZ substrates was performed using conventional photolithography and dry etching. The cubic InN channel layers were covered with $\mathrm{Au}$ (source and drain electrodes) and amorphous $\mathrm{HfO}_{2}$ (gate insulator).

images (Fig. 2a). It is well known that the quality of hetero-interfaces between gate insulators and semiconductors is critically important for the fabrication of high-performance FETs. Notably, the $\mathrm{HfO}_{2} /$ InN interface was found to be abrupt. We have confirmed the formation of an electron accumulation layer at the $\mathrm{HfO}_{2} / \mathrm{InN}$ interface by $\mathrm{x}$-ray photoelectron spectroscopy (see Supplementary Fig. S2). Finally, a $\mathrm{Au}$ film was deposited on the $\mathrm{HfO}_{2}$ and used as a gate electrode. The gate length $L_{\mathrm{G}}$ and width $W_{\mathrm{G}}$ of the FET varied from 5 to $50 \mu \mathrm{m}$.

\section{Discussion}

Figure 4a presents the output characteristics of the $\mathrm{HfO}_{2} / 2-\mathrm{nm}$ cubic-InN/YSZ FET. It can be seen that the current through the ultrathin InN can be well controlled by changing $V_{\mathrm{G}}$, which clearly has linear and saturation regions, indicating that the characteristics of this FET follow the conventional simple MOSFET theory. In addition, the FET operated in the depletion mode, indicating that the ultrathin cubic InN has a conduction channel for electrons even at zero bias. Figure $4 \mathrm{~b}$ shows the transfer characteristics of the 2-nmthick device. The on/off current ratio was $\sim 5 \times 10^{6}$. In this sample, the electrons are well depleted by the negative gate bias in the "off" state, and $I_{\mathrm{DS}}$ decreases to the order of pA. The "off"-state characteristics of this FET are better than $\mathrm{SiO}_{2}$ /InN/AIN FETs, whose "off" current is at the level of $\mathrm{nA}$ (ref. 6), which indicates that high-quality $\mathrm{HfO}_{2} / \mathrm{InN} / \mathrm{YSZ}$ interfaces are formed using the newly developed process. With the assumption that the electron density in the ultrathin cubic InN is $2.1 \times 10^{20} \mathrm{~cm}^{-3}$ (Ref. 14), the sheet electron density of that is $2.2 \times 10^{13} \mathrm{~cm}^{-2}$. This coincides with the fixed charge density in $\mathrm{HfO}_{2}\left(2.2 \times 10^{13} \mathrm{~cm}^{-2}\right.$ for dielectric constant $\varepsilon_{\mathrm{r}}$ of 16 and thickness of $20 \mathrm{~nm}$ ) with the gate voltage of $-5 \mathrm{~V}$. The fieldeffect mobility $\left(\mu_{\mathrm{FE}}\right)$ calculated using the relationship for the linear region $\left(I_{\mathrm{DS}}=\left(\mu_{\mathrm{FE}} C_{\mathrm{i}} W_{\mathrm{G}} / L_{\mathrm{G}}\right)\left(V_{\mathrm{GS}}-V_{\mathrm{TH}}\right) V_{\mathrm{DS}}\right)$ was $0.17 \mathrm{~cm}^{2} \mathrm{~V}^{-1} \mathrm{~s}^{-1}$. Figures $4 \mathrm{c}$ and $4 \mathrm{~d}$ show the output and transfer characteristics of the FET, respectively, with a channel thickness of $5 \mathrm{~nm}$. The on/off current ratio of this device is 37 , and the current density in "off"-state at $V_{\mathrm{DS}}=5 \mathrm{~V}$ and $V_{\mathrm{G}}=-10 \mathrm{~V}$ is $0.70 \mathrm{~mA} / \mathrm{mm}$. The drain current is approximately two orders of magnitude larger than that of the 2-nmthick InN FET, but the on/off ratio is considerably lowered. Maximum values for the $I_{\mathrm{DS}}$ and $\mu_{\mathrm{FE}}$ in this study are $61 \mathrm{~mA} / \mathrm{mm}$ and $58 \mathrm{~cm}^{2} \mathrm{~V}^{-1} \mathrm{~s}^{-1}$, respectively, which are obtained for a FET with channel thickness of $5 \mathrm{~nm}, W_{\mathrm{G}}$ of $20 \mu \mathrm{m}$, and $L_{\mathrm{G}}$ of $50 \mu \mathrm{m}$. We have also fabricated a FET with 3 -nm-thick InN channel ( $W_{\mathrm{G}}$ of $50 \mu \mathrm{m}$, and $L_{\mathrm{G}}$ of $\left.20 \mu \mathrm{m}\right)$, in which "on"-current density is $6.8 \mathrm{~mA} / \mathrm{mm}\left(V_{\mathrm{DS}}\right.$ $\left.=5 \mathrm{~V}, V_{\mathrm{G}}=2 \mathrm{~V}\right)$. When the same voltages of $V_{\mathrm{DS}}$ and $V_{\mathrm{G}}$ are applied in the FETs with channel thickness of 2 and $5 \mathrm{~nm}$, the drain current $I_{\mathrm{DS}}$ is 0.048 and $23 \mathrm{~mA} / \mathrm{mm}$, respectively. These values increase nonlinearly with an increase in the channel thickness. For comparison, we have fabricated a 4-nm-thick hexagonal InN FET on semi-insulating GaN substrates. The device characteristics are shown in Supplementary Fig. S3. The FET shows poor "off”-characteristics, probably due to a defective interface between InN and GaN. This result indicates that the abrupt cubic-InN/YSZ interface is responsible for the good characteristics of the ultrathin-InN-based FETs.
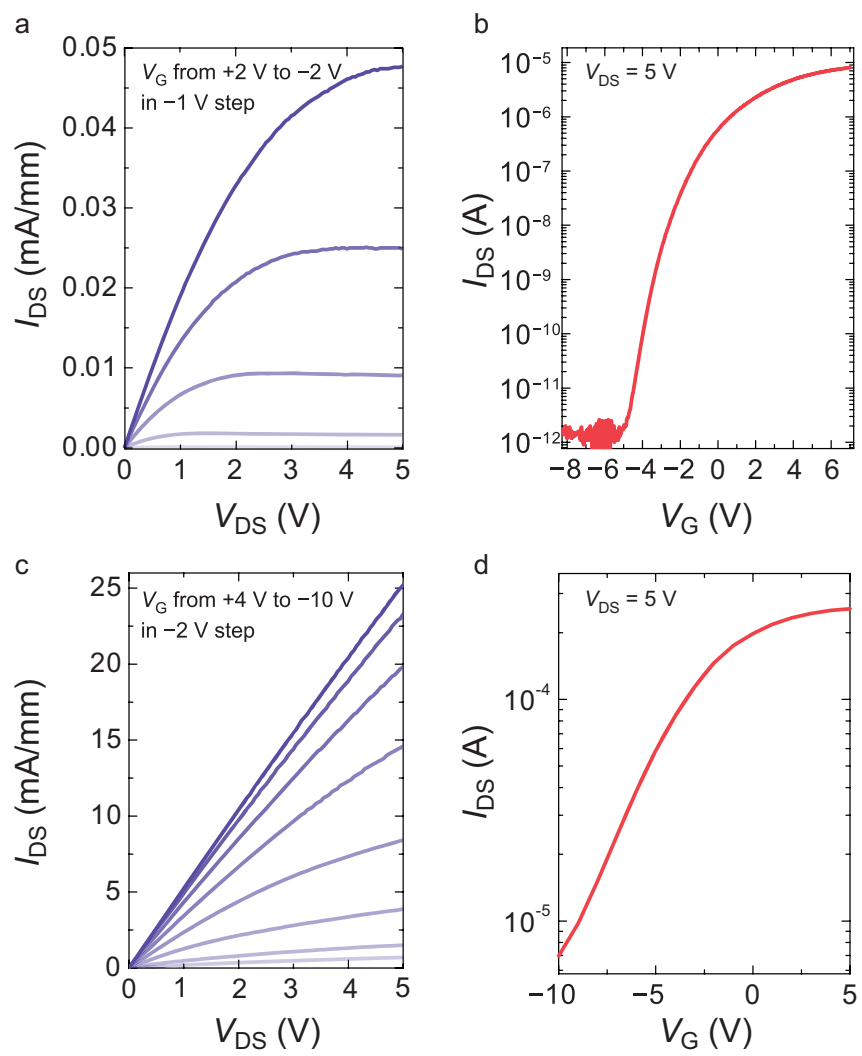

Figure $4 \mid$ (a) Output and (b) transfer characteristics of a cubic InN FET with a 2-nm-thick channel. The width and length of the gate are 50 and $20 \mu \mathrm{m}$, respectively. The $I_{\mathrm{DS}}$ on/off ratio at $V_{\mathrm{DS}}=5 \mathrm{~V}$ is $\sim 5 \times 10^{6}$. (c) Output and (d) transfer characteristics of a cubic InN FET with a 5-nmthick channel. The width and length of the gate are 10 and $50 \mu \mathrm{m}$, respectively. The field-effect mobility in the linear region is calculated to be $44 \mathrm{~cm}^{2} \mathrm{~V}^{-1} \mathrm{~s}^{-1}$. 
In conclusion, we have successfully fabricated FETs using ultrathin cubic InN channels. The FETs exhibited reasonable output and transfer characteristics that can be well explained by conventional FET theory. FETs fabricated with 2-nm-thick cubic InN exhibited high current on/off ratios of $\sim 5 \times 10^{6}$ and quite low off current densities at the $\mathrm{pA}$ level. The use of highly insulating YSZ substrates and the formation of abrupt $\mathrm{HfO}_{2} / \mathrm{InN} / \mathrm{YSZ}$ interfaces are responsible for the good off-state characteristics. Maximum values of $I_{\mathrm{DS}}$ and $\mu_{\mathrm{FE}}$ of the FETs fabricated with 5 -nm-thick InN are greater than $60 \mathrm{~mA} / \mathrm{mm}$ and $50 \mathrm{~cm}^{2} \mathrm{~V}^{-1} \mathrm{~s}^{-1}$, respectively. These results indicate that the use of ultrathin cubic InN is promising for the fabrication of future high-speed electron devices.

1. Tsen, K. T., Poweleit, C., Ferry, D. K., Lu, H. \& Schaff, W. J. Observation of large electron drift velocities in InN by ultrafast Raman spectroscopy. Appl. Phys. Lett. 86, 222103 (2005)

2. Kuzmik, J. \& Georgakilas, A. Proposal of High-electron mobility transistors with strained InN channel. IEEE Trans. Electron Devices 58, 720-724 (2011).

3. Lin, Y.-S. et al. High current density InN/AlN heterojunction field-effect transistor with a $\mathrm{SiN}_{x}$ gate dielectric layer. Appl. Phys. Lett. 90, 142111 (2007).

4. Ng, Y. F., Cao, Y. G., Xie, M. H., Wang, X. L. \& Tong, S. Y. Growth mode and strain evolution during InN growth on $\mathrm{GaN}(0001)$ by molecular-beam epitaxy. Appl. Phys. Lett. 81, 3960 (2002).

5. Wang, X. et al. High-electron-mobility InN layers grown by boundarytemperature-controlled epitaxy. Appl. Phys. Express 5, 015502 (2012).

6. Khan, N., Sedhain, A., Li, J., Lin, J. Y. \& Jiang, H. X. High mobility InN epilayers grown on AlN epilayer templates. Appl. Phys. Lett. 92, 172101 (2008).

7. Lu, C. J., Bendersky, L. A., Lu, H. \& Schaff, W. J. Threading dislocations in epitaxial InN thin films grown on (0001) sapphire with a GaN buffer layer. Appl. Phys. Lett. 83, 2817 (2003).

8. Wang, X., Che, S.-B., Ishitani, Y. \& Yoshikawa, A. Threading dislocations in Inpolar InN films and their effects on surface morphology and electrical properties. Appl. Phys. Lett. 90, 151901 (2007).
9. Piper, L. F. J., Veal, T. D., McConville, C. F., Lu, H. \& Schaff, W. J. Origin of the ntype conductivity of InN: The role of positively charged dislocations. Appl. Phys. Lett. 88, 252109 (2006).

10. Honke, T., Fujioka, H., Ohta, J. \& Oshima, M. InN epitaxial growths on Yttria stabilized zirconia (111) step substrates. J. Vac. Sci. Technol. A Vacuum, Surfaces, Film. 22, 2487 (2004)

11. Okubo, K., Kobayashi, A., Ohta, J., Oshima, M. \& Fujioka, H. Electron mobility of ultrathin InN on yttria-stabilized zirconia with two-dimensionally grown initial layers. Appl. Phys. Lett. 102, 022103 (2013).

12. Sato, K., Ohta, J., Inoue, S., Kobayashi, A. \& Fujioka, H. Room-temperature epitaxial growth of high quality AlN on $\mathrm{SiC}$ by pulsed sputtering deposition. Appl. Phys. Express 2, 011003 (2009).

13. Koblmüller, G., Gallinat, C. S. \& Speck, J. S. Surface kinetics and thermal instability of $\mathrm{N}$-face InN grown by plasma-assisted molecular beam epitaxy. J. Appl. Phys. 101, 083516 (2007).

14. Mahboob, I. et al. Origin of electron accumulation at wurtzite InN surfaces. Phys. Rev. B 69, 201307 (2004).

\section{Author contributions}

H.F. supervised the project. M.O. and K.O. performed film growth and device fabrication. A.K., J.O. and H.F. designed the experimental procedure. All the authors interpreted the data and wrote the paper.

\section{Additional information}

Supplementary information accompanies this paper at http://www.nature.com/ scientificreports

Competing financial interests: The authors declare no competing financial interests. How to cite this article: Oseki, M., Okubo, K., Kobayashi, A., Ohta, J. \& Fujioka, H. Field-effect transistors based on cubic indium nitride. Sci. Rep. 4, 3951; DOI:10.1038/ srep03951 (2014).

(c) (i) $\Theta$ This work is licensed under a Creative Commons Attribution-

( visit http://creativecommons.org/licenses/by-nc-nd/3.0 